\title{
Transformation design and nonlinear Hamiltonians
}

\author{
Thomas Brougham** Goce Chadzitaskos and Igor Jex \\ Department of Physics, FNSPE, Czech Technical University in Prague, Břehová 7, \\ 11519 Praha 1, Czech Republic.
}

October 16, 2018

\begin{abstract}
We study a class of nonlinear Hamiltonians, with applications in quantum optics. The interaction terms of these Hamiltonians are generated by taking a linear combination of powers of a simple 'beam splitter' Hamiltonian. The entanglement properties of the eigenstates are studied. Finally, we show how to use this class of Hamiltonians to perform special tasks such as conditional state swapping, which can be used to generate optical cat states and to sort photons. keywords: quantum optics, nonlinear optics, quantum information
\end{abstract}

\section{Introduction}

The consideration of nonlinear optical processes within quantum optics has led to the study of many important physical phenomena. These nonlinear optical processes have found many diverse applications, such as generating entangled photons using parametric down conversion [1] or creating optical routers using intensity dependent properties of an optical fibre [2, 3]. The growing field of quantum information has added to the interest in nonlinear optics. This is due to the fact that nonlinear optics can be used to perform certain operations that are not possible using only linear optics. For example, a class of nonlinear optical Hamiltonians, which have been used to model four wave mixers [4, 5, 6, 7, have been shown to allow one to prepare a macroscopically distinguishable superposition of quantum states.

In this paper we will consider a class of nonlinear Hamiltonians. The Hamiltonians will be functions (e.g. a polynomial) of a quadratic Hamiltonian, $\hat{H}_{0}$, that leads to linear differential equations in the field mode operators. Hamiltonians of this type have been used to describe optical beam splitters. The nonlinear Hamiltonians will thus commute with $\hat{H}_{0}$. Hence the task of obtaining the eigenvectors and eigenvalues of the nonlinear Hamiltonians will reduce to diagonalising the simple quadratic Hamiltonian. It will be shown that the eigenvalues and eigenvectors are related to a class of orthogonal Krawtchouk polynomials [8]. We thus establish that this particular class of nonlinear Hamiltonians are exactly solvable. The connection between orthogonal polynomials and nonlinear optical process has been studied previously and general results have been obtained $9,10,11$. In particular, the mathematical results that we present in section 2 is a special case of the general theory presented in 9 . In 9 the general method is described for solving two boson systems in quantum optics via of orthogonal polynomials systems. It is possible to study many of these problems using different mathematical techniques. In particular, beam splitters have bean studied extensively [12, 13, 14]. The benefit of using orthogonal polynomials, however, is that there exists extensive literature on their properties, which one can exploited for any given problem, see for example [8, 15].

The organization of the paper will be as follows. In section 2 we will diagonalise the quadratic Hamiltonian. This will be achieved by first showing the equivalence between diagonalising the

** Corresponding author. Email: thomas.brougham@gmail.com 
Hamiltonian and solving a particular recurrence relation. The solutions of the recurrence relation are the Krawtchouk polynomials. The properties of these polynomials will be used to obtain the spectrum of the Hamiltonian. In section 3 we study the entanglement properties of the energy eigenstates. The properties of the nonlinear Hamiltonians will be investigated in section 4. In particular we will show how the Hamiltonians can be used to perform a conditionally swap operation on the two modes, when the modes are both prepared with all the photons initially in one mode. We will also show how this enables one to prepare optical Schrödinger cat states. The dynamics of the nonlinear Hamiltonians is studied in further detail in section 5 , Finally, we discuss our results in section 6 .

\section{Diagonalising the quadratic Hamlitonian}

In this section we apply the method investigated in [9]. Suppose we have an electromagnetic field that has two modes 1 and 2. Associated with these modes are the creation and annihilation operators $\hat{a}_{j}^{\dagger}$ and $\hat{a}_{j}$, where $j=1,2$. These operators obey the standard canonical commutator relations $\left[\hat{a}_{j}, \hat{a}_{j}^{\dagger}\right]=\hat{1}$ and $\left[\hat{a}_{j}, \hat{a}_{k}\right]=0$, for $j \neq k$. Suppose further that the two modes interact. If this interaction is quadratic, then one possible form for the interaction Hamiltonian is

$$
\hat{H}_{0}=\gamma \hat{a}_{1}^{\dagger} \hat{a}_{2}+\gamma^{*} \hat{a}_{2}^{\dagger} \hat{a}_{1},
$$

where the free evolution terms are ignored 1 Hamiltonians of the form (1) are be used to describe beam splitters. A simple class of nonlinear interaction Hamiltonians can be constructed by introducing some nonlinear function $f(x)$ and forming a new Hamiltonian $\hat{H}=f\left(\hat{H}_{0}\right)$, where $\hat{H}_{0}$ is defined in equation (11). A simple example of this would be $f(x)$ a polynomial e.g.

$$
\hat{H}=\mu \hat{H}_{0}+\lambda\left(\hat{H}_{0}\right)^{2} .
$$

The nonlinear Hamiltonian described in equation (2) has been studied before in connection with various nonlinear optical phenomena, see for example [4, 6].

The quadratic Hamiltonian $\hat{H}_{0}$ conserves the total number of photons, i.e.

$$
\left[\hat{n}, \hat{H}_{0}\right]=0
$$

where $\hat{n}=\hat{n}_{1}+\hat{n}_{2}$. The class of nonlinear Hamiltonians $\hat{H}=f\left(\hat{H}_{0}\right)$, where $f(x)$ is some polynomial, will also conserve the total number of photons. By construction we see that $\left[\hat{H}_{0}, \hat{H}\right]=0$. We can thus obtain the eigenvectors of $\hat{H}$ by diagonalising $\hat{H}_{0}$. The fact that both Hamiltonians conserve the total photon number suggests that we should diagonalise the Hamiltonian (1) in subspaces with fixed numbers of photons. If we have a subspace where the total number of photons is constant and equals $M$, then the dimensions of this subspace will be $M+1$ and any state belonging to the subspace can be written in the form

$$
\left|\psi^{M}\right\rangle_{12}=\sum_{n=0}^{M} \xi_{n}|M-n, n\rangle_{12},
$$

where $|M-n, n\rangle_{12}$ represents the Fock state $|M-n\rangle_{1} \otimes|n\rangle_{2}$. Let $\left|E^{M}\right\rangle_{12}$ denote an eigenstate of (1), hence $\hat{H}_{0}\left|E^{M}\right\rangle_{12}=E\left|E^{M}\right\rangle_{12}$. For the sake of convenience we shall express the eigenstate in the following form

$$
\left|E^{M}\right\rangle_{12}=\sum_{n=0}^{M} c_{n}^{M} e^{-i n g}|M-n, n\rangle_{12},
$$

where $g=\arg (\gamma)$, i.e. $\gamma=|\gamma| e^{i g}$. Acting on equation (5) with $\hat{H}_{0}$ yields the expression

$$
\begin{aligned}
& \hat{H}_{0}\left|E^{M}\right\rangle_{12}=\gamma \sum_{n} \sqrt{n(M-n+1)} e^{-i n g} c_{n}^{M}|M-n+1, n-1\rangle_{12} \\
& +\gamma^{*} \sum_{n} \sqrt{(n+1)(M-n)} e^{-i n g} c_{n}^{M}|M-n, n+1\rangle_{12}=E\left|E^{M}\right\rangle_{12} .
\end{aligned}
$$

\footnotetext{
${ }^{1}$ One could imagine that we are working in an interaction picture.
} 
By re-arranging this expression one can show that the coefficients $c_{n}^{M}$, obey the following three term recurrence relation

$$
\frac{E}{|\gamma|} c_{n}^{M}=\sqrt{(n+1)(M-n)} c_{n+1}^{M}+\sqrt{n(M-n+1)} c_{n-1}^{M} .
$$

The recurrence relation (7) can be solved to find both the eigenvalues and the coefficients that appear in the Fock basis expansion of the energy eigenvectors. The mathematical properties of the recurrence relation (7) enable us to determine many properties of the eigenvectors and eigenvalues of energy. For example, if equation (5) is an eigenvector of $\hat{H}_{0}$ corresponding to the eigenvalue $E$, then so is the vector $\left|-E^{M}\right\rangle_{12}=\sum_{n}(-1)^{n} \exp (-i n g) c_{n}^{M}|M-n, n\rangle_{12}$, which corresponds to the eigenvalue $-E$. The proof of this fact follows simply by replacing the terms $c_{n}^{M}$, in (7), with $(-1)^{n} c_{n}^{M}$. We thus see that for every positive eigenvalue of energy there must exist a corresponding negative energy eigenvalue.

The coefficients $c_{n}^{M}$ will be functions of $E$. If we formally solve the recurrence relation (77), then we find that $c_{n}^{M}(E)$ is a polynomial of degree $n$, in the variable $E$. The fact that the total number of photons is $M$ means that $c_{M+1}^{M}(E)=0$, which means that the roots of the polynomial $c_{M+1}^{M}(E)$ correspond to the eigenvalues of (11). To help explain the last fact consider the matrix representation of equation (7)

$$
\frac{E}{|\gamma|}\left(\begin{array}{c}
c_{0}^{M} \\
c_{1}^{M} \\
\vdots \\
c_{M}^{M}
\end{array}\right)=\left(\begin{array}{cccccc}
0 & \sqrt{M} & 0 & 0 & \ldots & 0 \\
\sqrt{M} & 0 & \sqrt{2(M-1)} & 0 & \ldots & 0 \\
\vdots & \vdots & \vdots & \vdots & \vdots & \vdots \\
0 & \ldots & \ldots & \sqrt{M} & 0 &
\end{array}\right)\left(\begin{array}{c}
c_{0}^{M} \\
c_{1}^{M} \\
\vdots \\
c_{M}^{M}
\end{array}\right)+\left(\begin{array}{c}
0 \\
0 \\
\vdots \\
\phi_{M+1} c_{M+1}^{M}
\end{array}\right),
$$

where $\phi_{M+1}$ is the term that we would find in front of $c_{M+1}^{M}$ in the recurrence relation (11). It can be seen that if $c_{M+1}^{M}(E)=0$, then the column vector $\mathbf{c}^{M}$ will be an eigenvector of the Hamiltonian, corresponding to the eigenvalue $E /|\gamma|$. An important point to note about equation (17) is that the factors that multiply both $c_{n+1}^{M}$ and $c_{n-1}^{M}$ are positive. This fact enables us to make use of a theorem of Favard's [17. For a recurrence relation of the form

$$
x P_{n}(x)=b_{n+1} P_{n+1}(x)+b_{n-1} P_{n-1}(x),
$$

where the sequence $\left\{b_{n}\right\}$ is non-negative, there exists a linear functional $\mathcal{L}$, which has the following properties

$$
\begin{aligned}
\mathcal{L}[1] & =1, \\
\mathcal{L}\left[P_{m}(x) P_{n}(x)\right] & =0 \text { for } m \neq n, \\
\mathcal{L}\left[P_{n}^{2}(x)\right] & \neq 0 .
\end{aligned}
$$

Applying this theorem to (7) leads to the conclusion that the set of polynomials $\left\{c_{n}^{M}(x)\right\}$, will be orthogonal to each other with respect to the linear functional $\mathcal{L}$. A standard property of orthogonal polynomials is that the roots of an orthogonal polynomial are distinct [16. This last result together with the fact that the eigenvalues of $\hat{H}_{0}$ are the roots of the polynomial $c_{M+1}^{M}(E)=0$, implies that the eigenvalues of $\hat{H}_{0}$ are nondegenerate, when we are confined to the $M$ photon subspace 2 . There is an extensive literature on orthogonal polynomials and their properties [8, 15, 16]. One important example are the Krawtchouk polynomials, which obey the following three term recurrence relation $[8]$

$$
\begin{aligned}
x K_{n}(x ; p, M)= & \sqrt{p(1-p)(n+1)(M-n)} K_{n+1}(x ; p, M)+(p[M-n]+n[1-p]) K_{n}(x ; p, M)+ \\
& +\sqrt{p(1-p) n(M-n+1)} K_{n-1}(x ; p, M),
\end{aligned}
$$

where $0<p<1$. If we set $p=1 / 2,2 x-M=E /|\gamma|$ and $K_{n}(x ; 1 / 2, M)=c_{n}^{M}(E)$, then equation (11) reduce to equation (7). The coefficients $c_{n}^{M}(E)$ are thus Krawtchouk polynomials.

\footnotetext{
${ }^{2}$ The spectrum of $\hat{H}_{0}$ on the whole Hilbert space will have degeneracies due to the fact that the spectrum of $\hat{H}_{0}$ on the different subspace is not disjointed and thus can share eigenvalues.
} 
The form of the Krawtchouk polynomials, for this choice of parameters, is given by

$$
K_{n}(x ; 1 / 2, M)=(-1)^{n} K_{0}(x ; 1 / 2, M) \sqrt{\frac{M !}{n !(M-n) !}} \sum_{k=0}^{n} \frac{(-n)_{k}(-x)_{k}}{(-M)_{k} k !} 2^{k},
$$

where $(y)_{k}=y(y+1)(y+2) \ldots(y+k-1)$. The roots of the Krawtchouk polynomials can be determined using (12). It can easily be shown that the roots of $K_{M+1}(x ; 1 / 2, M)=0$ are $x=0,1,2, \ldots, M$, which implies that the energy eigenvalues are

$$
E=-M|\gamma|,(2-M)|\gamma|,(4-M)|\gamma|, \ldots, M|\gamma|
$$

By diagonalising the Hamiltonian (1) we have solved the system's dynamics and can analyze relevant quantum effects.

\section{Photon distribution and entanglement properties of the energy eigenstates}

In the previous section we found the analytic solution of equation (7), which in turn gives us the form of the eigenvectors of the Hamiltonian (11). Nevertheless, it is informative to give some simple examples of the eigenvectors of (11). For the simplest case, when $M=1$, the eigenvalues are just $E= \pm|\gamma|$ and the eigenvectors take the form $|E \pm\rangle=(|10\rangle \pm \exp (-i g)|01\rangle) / \sqrt{2}$. When $M=2$, we find that $E= \pm 2|\gamma|, 0$ and

$$
\begin{aligned}
|E= \pm 2| \gamma|\rangle & =\frac{1}{2}\left(|20\rangle \pm \sqrt{2} e^{-i g}|11\rangle+e^{-2 i g}|02\rangle\right), \\
|E=0\rangle & =\frac{1}{\sqrt{2}}\left(|20\rangle-e^{-2 i g}|02\rangle\right) .
\end{aligned}
$$

As $M$ increases the form of the eigenvectors will generally become more complicated. One situation, however, where the eigenvectors have a simple form is when $E=M|\gamma|$, i.e for the eigenvectors corresponding to the maximum eigenvalue of $\hat{H}_{0}$. In this case we find

$$
\begin{gathered}
c_{n}^{M}=K_{n}(M ; 1 / 2, M)=(-1)^{n} c_{0}^{M} \sqrt{\frac{M !}{n !(M-n) !}} \sum_{k=0}^{n} \frac{(-n)_{k}}{k !} 2^{k} \\
=(-1)^{n} c_{0}^{M} \sqrt{\frac{M !}{n !(M-n) !}} \sum_{k=0}^{n} \frac{n !}{k !(n-k) !}(-2)^{k}=c_{0}^{M} \sqrt{\frac{M !}{n !(M-n) !}} .
\end{gathered}
$$

If $c_{0}^{M}$ is chosen to be $2^{-M / 2}$, then $\sum_{n}\left|c_{n}^{M}(M|\gamma|)\right|^{2}=1$. A consequence of this result is that the photon probability distribution, $\left|c_{n}^{M}\right|^{2}$, will be a binomial distribution when $E=M|\gamma|$. In the limit of large $M,\left|c_{n}^{M}(M|\gamma|)\right|^{2}$ can be approximated by a Gaussian.

The probability distribution $\left|c_{n}^{M}\right|^{2}$, of finding $n$ photons in the second mode, depends only on $|E|$. By this we mean that $\left|c_{n}^{M}(E)\right|^{2}=\left|c_{n}^{M}(-E)\right|^{2}$. This follows directly from the result in section 2 that $c_{n}^{M}(-E)=(-1)^{n} c_{n}^{M}(E)$, which can be verified using the recurrence relation (7). The behaviour of the probability distributions $\left|c_{n}^{M}(E)\right|^{2}$, for $|E|<M|\gamma|$, can be studied by numerically evaluating (12). Figure 1 shows $\left|c_{n}^{M}\right|^{2}$ plotted with $M=38$, for the four largest eigenvalues of $\hat{H}_{0}$. We see that each time $E$ is decreased by $2|\gamma|$, an additional peak appears in the probability distribution. Numerical investigations show that this behaviour is a generic feature and occurs for each value of $M$. Figure 1 shows that the probability distribution has the symmetry, $\left|c_{n}^{M}\right|^{2}=\left|c_{M-n}^{M}\right|^{2}$. This means that the probability of finding $n$ photons in the first mode is the same as finding $n$ photons in the second mode. This result is physically reasonable due to the symmetric nature of the Hamiltonian (10), with regards to the two modes.

If we calculate the explicit forms for the energy eigenvectors, then we see that the two modes are entangled, i.e they cannot be express in a factorised form. Entanglement is one of the defining characteristics of quantum mechanics and has great importance in the foundations of quantum 

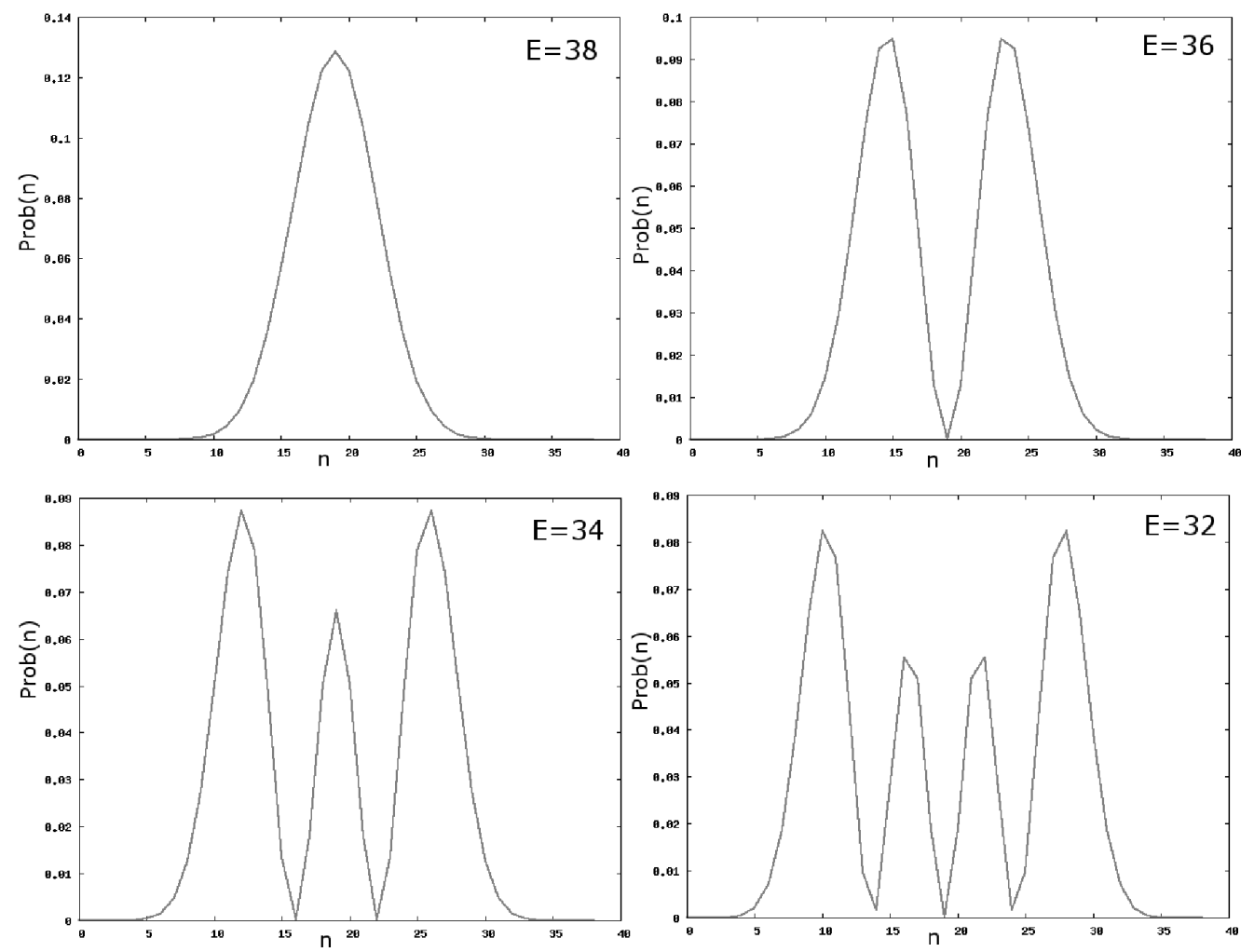

Figure 1: Four different plots of the probability distribution for finding $n$ photons in the second mode. Each plot is for the situation where $M=38$ and $|\gamma|=1$, however in each successive plot the energy is decreased by $2|\gamma|$. 


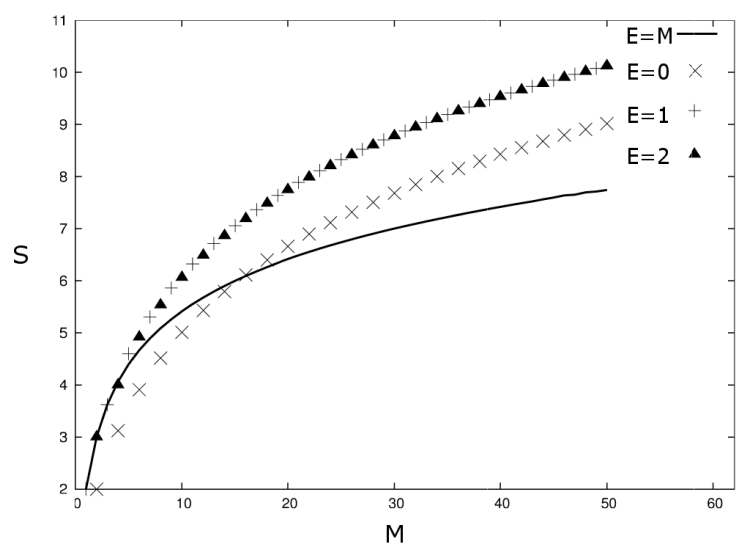

Figure 2: A plot of the entanglement, $S_{\text {ent }}$, against the number of photons $M$, for different values of energy $E$, with $\gamma=1$.

mechanics and in the field quantum information [18. For this reason it is of interest to quantify the entanglement present in the energy eigenstates. A common approach to quantifying the entanglement in a bipartite pure state is to calculate the von Neumann entropy in the reduced state [18, 19, 20]. The von Neumann entropy of a density operator $\hat{\rho}$ is defined to be

$$
S[\hat{\rho}]=-\operatorname{Tr}[\hat{\rho} \log (\hat{\rho})],
$$

where the logarithms are taken to the base 2 . The reduced states of the bipartite state $|\psi\rangle_{12}$ are define to be $\hat{\rho}_{1}=\operatorname{Tr}_{2}[|\psi\rangle\langle\psi|]$ and $\hat{\rho}_{2}=\operatorname{Tr}_{1}[|\psi\rangle\langle\psi|]$, where $\operatorname{Tr}_{j}$ denotes the partial trace over the subsystem $j$. We thus take use the following quantity to measure the entanglement [19]

$$
S_{\text {ent }}(|\psi\rangle\langle\psi|)=2 S\left(\hat{\rho}_{1}\right)=2 S\left(\hat{\rho}_{2}\right) .
$$

$S_{\text {ent }}(|\psi\rangle\langle\psi|)=0$ when our bipartite state is separable. In addition to this, we can see that equation (17) assumes its maximum value when our bipartite state is a maximally entangled state, i.e. the reduced states are proportional to the identity operator. If we express the energy eigenvectors in the form given in equation (5), then the eigenstates are automatically in the Schmidt form [18, where the basis states $\left\{|M-n, n\rangle_{12}\right\}$ now act as our Schmidt basis. From this observation is is straightforward to show that $S_{\text {ent }}$ equals twice the entropy of the probability distribution $\left|c_{n}^{M}(E)\right|^{2}$, i.e

$$
S_{\text {ent }}=2 S\left(\left|c_{n}^{M}\right|^{2}\right)=-2 \sum_{n}\left|c_{n}^{M}\right|^{2} \log \left(\left|c_{n}^{M}\right|^{2}\right),
$$

where the logarithms are taken to the base 2 . We can now evaluate $S_{\text {ent }}$ as a function of the total number of photons $M$. Figure 2 shows $S_{\text {ent }}$ plotted against $M$, with the energy $E$ either fixed or set equal to the largest possible value given the choice of $M$. We see that as $M$ increases so does $S_{\text {ent }}$. The fact that the entanglement increases with $M$ is not surprising as the dimensions of our systems Hilbert space are $M+1$. Increasing the number of photons thus gives us more terms in the Schmidt decomposition of the eigenstates $\left|E^{M}\right\rangle_{12}$. We can also see that the zero eigenstate, i.e. the state $\left|E^{M}\right\rangle_{12}$, with $E=0$, is less entangled than the states with $E=1$ or $E=2$. By examining equation (77) is can be seen that for $E=0$, we would require that every term $c_{n}^{M}$ equal zero for $n$ odd. The zero eigenvector thus has roughly half the number of Schmidt terms as the other eigenvectors do. Consequently, the zero eigenvector is not as entangled as the eigenvectors that correspond to $E \neq 0$.

It is interesting to see how the entanglement changes as we vary $E$, with the total number of photons now fixed. Figure 3 shows $S_{\text {ent }}$ plotted against the eigen-energy, $E$, for several different values of $M$. It can be seen that when $E$ is large, the entropy decreases. The fact that there 


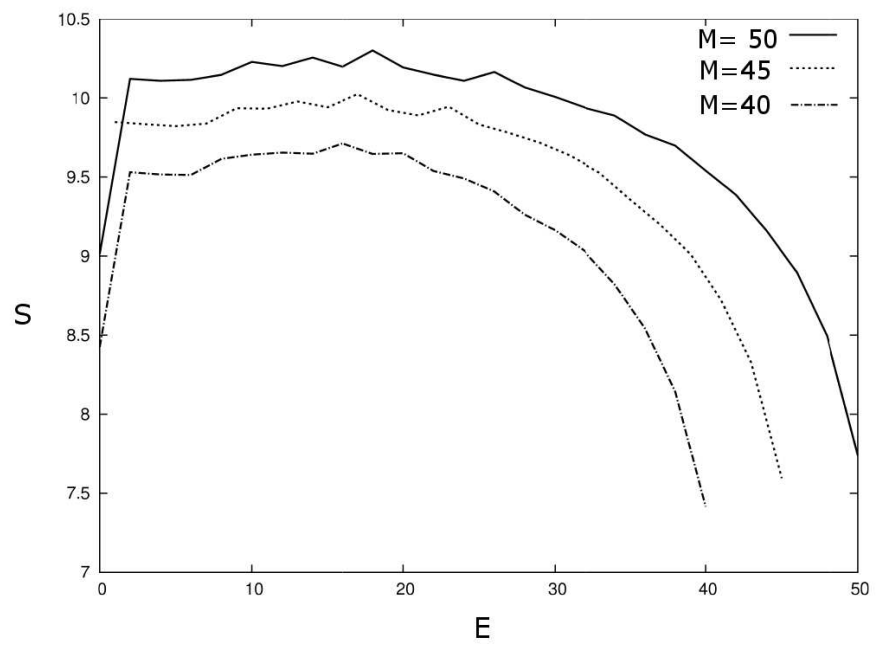

Figure 3: Entanglement, $S_{\text {ent }}$, plotted against the energy $E$, for different values of the photon number $M$.

is a general trend for the entanglement to decrease as $E$ increases can be explained by looking at how the probability distribution, $\left|c_{n}^{M}(E)\right|^{2}$, changes when $E$ varies. Figure 1 shows that as we decrease $E$ additionally peaks appear in the probability distribution. This means that the probability becomes more spread out and thus the entropy increases. Thinking in terms of correlations we see that for $E$ equal to $M|\gamma|$, we have a single maximum in the probability distribution $\left|c_{n}^{M}\right|^{2}$. The Schmidt basis states $|M-n, n\rangle_{12}$ will only have coefficients with large values for the states with $n$ close to $M / 2$. When $E$ is decreased we obtain several maxima in the probability distribution $\left|c_{n}^{M}(E)\right|^{2}$ and thus we have more Schmidt basis states with larger coefficients and hence the entanglement increases.

An interesting feature of figure 3 is that there are points where the entanglement increase slightly as $E$ increases. One obvious example of this is when $M$ is even and $E$ increases from zero to $2(\gamma=1$ in figure 3). In this case the increase is due to the anomalous nature of the zero energy eigenvector, i.e it has roughly half the number of nonzero Schmidt coefficients as the other energy eigenvectors. The other instances when the entanglement increase occur due to the fact that $P_{n}^{M}(E)$ can sometimes broaden when $E$ increases. Sometimes this broadening will compensate for the loss of peaks in $P_{n}^{M}(E)$ and thus the probability distribution is more spread out, which leads to a slight increase in the entropy.

\section{Nonlinear Hamiltonians and conditional state swapping}

In section 2 we obtained the eigenvalues and eigenvectors corresponding to the quadratic Hamiltonian (11). These results can now be used to determine the properties of a certain class of nonlinear Hamiltonians, which are constructed from $\hat{H}_{0}$ [an example of this is given in equation (2)].

Let $f(y)$ be a polynomial in the variable $y$, it is clear that $\left[\hat{H}_{0}, f\left(\hat{H}_{0}\right)\right]=0$, and thus $f\left(\hat{H}_{0}\right)\left|E^{M}\right\rangle_{12}=f(E)\left|E^{M}\right\rangle_{12}$. Similarly, the operator $\hat{n}=\hat{n}_{1}+\hat{n}_{2}$, obeys $\left[\hat{n}, \hat{H}_{0}\right]=0$ and thus $f(\hat{n})\left|E^{M}\right\rangle_{12}=f(M)\left|E^{M}\right\rangle_{12}$. From this we see that the general Hamiltonian

$$
\hat{H}=\sum_{k} \omega_{k} \hat{n}^{k}+\alpha_{k}\left(\hat{H}_{0}\right)^{k}
$$

can be diagonalised using the eigenvectors $\left\{\left|E^{M}\right\rangle_{12}\right\}$. We should note that the spectrum of equation (19) will generally be degenerate and thus we can construct several different sets of eigenvectors for the Hamiltonian. One important practical consideration for our nonlinear 
Hamiltonians is that the magnitude of the terms $\alpha_{k}$ should decrease as $k$ increases. In practice, if we want the higher order terms in our Hamiltonian to give an non-negligible contribution to the evolution of our system, we would have to increase the intensity of our light beams. This would correspond to $M$ being large. Even when we increase the intensity of the optical beams that enter the nonlinear media, it will still be difficult to excite higher order terms. For this reason we shall look only at examples were $\hat{H}$ is at most quadratic in $\hat{H}_{0}$, i.e. Hamiltonian with a form given in equation (2).

Using our previous results we can easily determine the time evolution of a system described by a Hamiltonian of the form given in equation (19). If at time $t=0$ the system is prepared in the state $\left|\psi_{0}\right\rangle_{12}$, then at a later time $t$, it will be in the state $\left|\psi_{t}\right\rangle_{12}=\exp (-i \hat{H} t)\left|\psi_{0}\right\rangle_{12}$, where $\hbar=1$. The problem of determining the evolution thus reduces to expressing the state $\left|\psi_{0}\right\rangle_{12}$ in terms of the basis $\left\{\left|E^{M}\right\rangle_{12}\right\}$. In quantum optics, states are often expressed in terms of the Fock basis. It is thus essential to be able to express a given Fock state in terms of the energy eigenvectors. This can be achieved using the completeness relation, $\sum_{k}\left|E_{k}\right\rangle\left\langle E_{k}\right|=\hat{1}$, which enables us to obtain that

$$
|M-n, n\rangle_{12}=\sum_{k}{ }_{12}\left\langle E_{k} \mid M-n, n\right\rangle_{12}\left|E_{k}\right\rangle_{12}=e^{i n g} \sum_{k} c_{n}^{M}\left(E_{k}\right)\left|E_{k}\right\rangle_{12} .
$$

We now give examples of how the preceding theory can be used to construct Hamiltonians that perform specific tasks. In order to simplify the following calculations we set $\gamma$ real. Suppose we have the Fock state $|M, 0\rangle_{12}$, which we want to transform to the state $|0, M\rangle_{12}$, i.e. we swap the two modes. From equation (20) it is clear that if we want to perform this task, then we must change the coefficient $c_{0}^{M}\left(E_{k}\right)$ to $c_{M}^{M}\left(E_{K}\right)$. Using equation (12) together with some simple algebra leads to the result that

$$
c_{M}^{M}\left(E_{k}\right)=(-1)^{M+x_{k}} c_{0}^{M}\left(E_{k}\right),
$$

where $x_{k}=\left(E_{k}+M|\gamma|\right) /(2|\gamma|)$. This result means that when $M+x_{k}$ is odd, $c_{M}^{M}\left(E_{k}\right)=$ $-C_{0}^{M}\left(E_{k}\right)$, while for $M+x_{k}$ even $c_{M}^{M}\left(E_{k}\right)=c_{0}^{M}\left(E_{k}\right)$. Suppose that we wish to construct a Hamiltonian that will peform the swap operation $|M, 0\rangle_{12} \rightarrow|0, M\rangle_{12}$ for all values of $M$. There is an infinity of different Hamiltonians, which achieve this task. An example of a simple Hamiltonian that performs the swap operation is

$$
\hat{H}=\frac{\pi}{2|\gamma| \tau}\left(3|\gamma| \hat{n}+\hat{H}_{0}\right)
$$

where $\exp (-i \hat{H} \tau)|M, 0\rangle_{12}=|0, M\rangle_{12}$. This Hamiltonian is linear in $\hat{H}_{0}$, we could, however, construct alternative nonlinear Hamiltonians that perform the swap operation. An interesting point to note is that if we had some state $|\psi\rangle=\sum_{n} \xi_{n}|n\rangle$, then the Hamiltonian described in equation (22) can be used to enact the transformation $|\psi\rangle_{1}|0\rangle_{2} \rightarrow|0\rangle_{1}|\psi\rangle_{2}$, i.e. $\exp (-i \hat{H} \tau)|\psi\rangle_{1}|0\rangle_{2}=|0\rangle_{1}|\psi\rangle_{2}$.

In the previous example the swap operation was insensitive to the total number of photons. It is, however, possible to design Hamiltonians that will perform a swap operation only if the number of photons is either even or odd. Suppose now that we want the swap operation to occur only if the total number of photons is even, i.e $|M, 0\rangle_{12} \rightarrow|0, M\rangle_{12}$ only when $M$ is even. Let $\epsilon_{x}^{M}$ denote the energy eigenvalue of our nonlinear Hamiltonian, corresponding to the eigenvector $\left|E_{x}^{M}\right\rangle_{12}$. In order to perform the swap at time $t=\tau$ we require that $\exp \left(-i \epsilon_{x}^{M} \tau\right)=(-1)^{M+x}$, but only when $M$ is even. A Hamiltonian that achieves this is

$$
\hat{H}=\frac{\pi}{|\gamma| \tau}\left(|\gamma| \hat{n}+\frac{|\gamma|}{4} \hat{n}^{2}+\frac{1}{4|\gamma|} \hat{H}_{0}^{2}\right) .
$$

One can easily verify that

$$
\exp (-i \hat{H} \tau)|M, 0\rangle_{12}=\left\{\begin{array}{l}
|0, M\rangle_{12}, \text { for } M \text { even } \\
i|M, 0\rangle_{12}, \text { for } M \text { odd }
\end{array}\right.
$$


The Hamiltonian (23) thus has the property that for a state $|\psi\rangle$, the transformation $|\psi\rangle_{1}|0\rangle_{2} \rightarrow$ $|0\rangle_{1}|\psi\rangle_{2}$ can only be realized if $|\psi\rangle$ can be expressed as a superposition of Fock states consisting of an even number of photons.

We have described how the Hamiltonian given in equation (23), can be used to perform a conditional swap operation. The Hamiltonian can also be used to perform other useful tasks, such as preparing two optical cat states form a single coherent state. An optical cat is a state of the form $K(|\alpha\rangle+|-\alpha\rangle)$, where $K$ is a normalisation constant and $| \pm \alpha\rangle$ are coherent states [?]. The significance of these states can be seen by recalling that when $|\alpha|$ is large, coherent state behave like a classical monochromatic optical fields. Cat states are thus a superposition of two classical object:3. The Fock state representation of a coherent state is 22

$$
|\alpha\rangle=\exp \left(-\frac{|\alpha|^{2}}{2}\right) \sum_{n=0}^{\infty} \frac{\alpha^{n}}{\sqrt{n !}}|n\rangle
$$

where $\alpha$ is a complex number. If we take our initial state to be $|\alpha\rangle_{1}|0\rangle_{2}$, then using the Hamiltonian (23) will yield the following evolution

$$
\exp (-i \hat{H} \tau)|\alpha\rangle_{1}|0\rangle_{2}=|0\rangle_{1}\left(\frac{|\alpha\rangle_{2}+|-\alpha\rangle_{2}}{2}\right)+i\left(\frac{|\alpha\rangle_{1}-|-\alpha\rangle_{1}}{2}\right)|0\rangle_{2}
$$

We have thus obtained a superposition of two optical cat states. Theoretical schemes for obtaining a superposition of two optical cat states have been proposed previously [4, 6, 7]. In each of the earlier schemes, the Hamiltonians that were used were different from that given in equation (23).

As we have shown, it is possible to construct Hamiltonians that perform a swap operation conditioned on whether the number of photons is even. It is also interesting to consider a swap operation conditioned on other factors. For instance, we may want to not swap the state if it has a particular number of photons, while allowing the swap to occur if the number of photons differs by one. We now give the form of a Hamiltonian that performs this task. As before, let $\epsilon_{x}^{M}$ denote the energy of the nonlinear Hamiltonian. We require that $\exp \left(-i \epsilon_{x}^{M} \tau\right)=(-1)^{M+x}$ if $M=N \pm 1$, but that $\exp \left(-i \epsilon_{x}^{M} \tau\right) \neq(-1)^{M+x}$ for $M=N$. It can easily be verified that the following Hamiltonian satisfies the stated conditions

$$
\hat{H}=\frac{\pi}{2|\gamma| \tau}\left(3|\gamma| \hat{n}^{2}+\hat{n} \hat{H}_{0}-3 N|\gamma| \hat{n}-N \hat{H}_{0}\right) .
$$

This leads to the following dynamics, $\exp (-i \hat{H} \tau)|N, 0\rangle_{12}=|N, 0\rangle_{12}$, while $\exp (-i \hat{H} \tau) \mid N \pm$ $1,0\rangle_{12}=|0, N \pm 1\rangle_{12}$. It is straightforward to modify the Hamiltonian given in equation (27) for situations where we want to swap states other than $|N \pm 1,0\rangle_{12}$. An example of this is when we want to swap states such as $|N \pm 2,0\rangle_{12}$. To achieve this we can simply multiply equation (27) by a factor of $1 / 2$, i.e. we take our Hamiltonian to be $\hat{H} / 2$. We shall now show how one can use the Hamiltonian (27) together with our previous nonlinear Hamltonians, so as to discriminate between photon number states.

Suppose we have four modes that are initially prepared in the state

$$
|M\rangle_{1}|0\rangle_{2}|0\rangle_{3}|0\rangle_{4}
$$

where $M \leq 4$. An important problem to address is whether we can determining the value of $M$. The majority of currently available photon detectors are not capable of reliably discriminating between different photon number states. For this reason it would be interesting if we could act on the state (28), so that the final position of the mode that is not in the vacuum is dependent on the number of photons. In particular, we could arrange for the state to evolve such that the $M$-th mode contains $M$ photons, while the other modes are in the vacuum state. We shall now outline a scheme that enables one to performs this task.

\footnotetext{
${ }^{3}$ The name 'cat state' is a reference to the thought experiment developed by Schrödinger [23].
} 
At time $t=0$ our system is prepared in the state (28). We arrange for modes 1 and 2 to interact in a manner governed by the Hamiltonian (23). This means that at time $\tau$ the photons will have been transferred to the second modes if $M$ is even, or remain in the first mode for $M$ odd. We now arrange for the first and third modes to be coupled via a Hamiltonian of the form $\hat{H} / 2$, where $\hat{H}$ is given by equation (27) and where the $N$ in equation (27) equals 1 . This will result in the first and third mode being swapped when we have three photon. At the same time we shall also couple the second and fourth modes using a Hamiltonian of the form $\hat{H} / 2$, where $\hat{H}$ is given by equation (27), with $N=2$. This will result in the second and fourth modes being swapped if the number of photons is four. The effect of coupling the modes in the manner that we have described is that our system will be left in the state

$$
\begin{array}{r}
i|1\rangle_{1}|0\rangle_{2}|0\rangle_{3}|0\rangle_{4}, \text { for } M=1 \\
|0\rangle_{1}|2\rangle_{2}|0\rangle_{3}|0\rangle_{4}, \text { for } M=2, \\
|0\rangle_{1}|0\rangle_{2}|3\rangle_{3}|0\rangle_{4}, \text { for } M=3, \\
|0\rangle_{1}|0\rangle_{2}|0\rangle_{3}|4\rangle_{4}, \text { for } M=4
\end{array}
$$

We could now put photon detectors at each mode so as to determine which mode contains photons. This in turn would allow us to determine the number of photons that we have. Alternatively we could perform further operations on the individual modes. In addition to this, the above scheme can be used to prepare multiphoton entangled states, provided we can prepare a superposition of a finite number of Fock states, in a single modes. For example, we could transform the separable state $(a|1\rangle+b|2\rangle)|0\rangle$ into the entangled state $a|10\rangle+b|02\rangle$. The various procedures that we have outlined could find applications in quantum information processing and quantum control.

\section{Dynamics of the nonlinear Hamiltonians}

In the previous section we constructed nonlinear Hamiltonians that allowed use to perform certain types of operations on the two coupled modes. In this section we will investigate further the dynamics induced by the three Hamiltonians given by (22), (23) and (27). A natural quantity to study is the number of photons in each mode. For this reason we shall investigate how the expectation value for the number of photons in the second mode changes, i.e. $\left\langle\hat{n}_{2}\right\rangle$. The initial state of our system will be taken to be $|M, 0\rangle_{12}$, and thus $\left\langle\hat{n}_{2}\right\rangle=0$ at time zero.

We shall now study the dynamics associated with the quadratic Hamiltonian (22). In figure 4 we see the expectation value $\left\langle\hat{n}_{2}\right\rangle$ plotted as a function of time, for the initial states $|10,0\rangle_{12}$ and $|11,0\rangle_{12}$, with $\gamma=\tau=1$. The expectation value oscillates with period of two (i.e. $2 \tau$ ), the period is thus independent of the number of photons. Another interesting feature of figure 4 is that at time $t=1,\left\langle\hat{n}_{2}\right\rangle=M$, which is consistent with the state having been transformed to $|0, M\rangle_{12}$ at that time. The oscillatory dynamics shown in figure 4 is also observed for other values of $M$, and does not depend on whether $M$ is even or odd. One final interesting feature of the Hamiltonian (22) is that we obtain oscillations for different choices of the initial state. For example, if the state was initially $|M-n, n\rangle$, then we would still observe oscillations of $\left\langle\hat{n}_{2}\right\rangle$, with period $2 \tau$. The minimum and maximum values of $\left\langle\hat{n}_{2}\right\rangle$ would, however, respectively change to $\left\langle\hat{n}_{2}\right\rangle=n$ at $t=0$ and $\left\langle\hat{n}_{2}\right\rangle=M-n$ at $t=\tau$. Such behaviour is consistent with the Hamiltonian (22) being able to swap the two modes.

The Hamiltonian (23) was designed to perform the transformation described in equation (24). This selective transformation has similarities to the behaviour of a two mode nonlinear directional coupler [2, 3]. This is an optical device which consists of two optical fibers that are coupled in such a way that the dynamics depends on the intensity of the light [3]. One obvious difference between these two nonlinear systems is that the conditional dynamics associated with the Hamiltonian (23), dependent on whether the number of photons is even or odd and not on how many photons there are. Figure 5 shows a plot of the expectation value $\left\langle\hat{n}_{2}\right\rangle$, against the time, for the initial states $|10,0\rangle_{12}$ and $|11,0\rangle_{12}$. We see that the dynamics is very different for the two different initial states. In particular, $\left\langle\hat{n}_{2}\right\rangle$ never exceeds the value 5.5, for the case when 


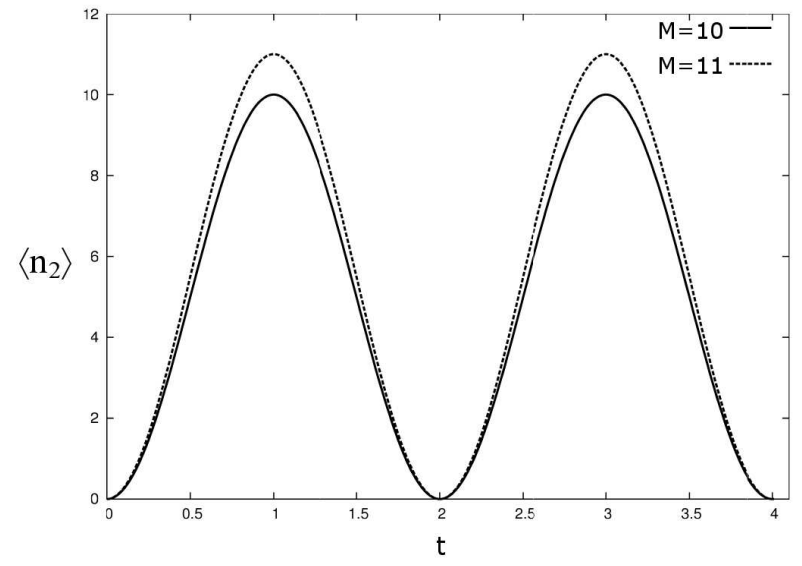

Figure 4: A plot of the expectation value $\left\langle\hat{n}_{2}\right\rangle$, against time, with $\gamma=\tau=1$ and where the initial state is $|M, 0\rangle_{12}$.

$M=11$. This is in contrast to the situation for $M=10$, where we find that $\left\langle\hat{n}_{2}\right\rangle=10$ at $t=1$ (i.e. $t=\tau$ ). In both cases we see that the behaviour of $\left\langle\hat{n}_{2}\right\rangle$ is periodic, with period $t=2 \tau=2$. When $M=10$, one can observe that the expectation value $\left\langle\hat{n}_{2}\right\rangle$ temporarily plateaus at the value of 5 , which is half the total number of photons.

It is interesting to investigate the dynamics of the Hamiltonian (24) for initial states, which have photons in both modes, i.e $|M-n, n\rangle_{12}$, with $n \neq 0$. Figure (6) show $\left\langle\hat{n}_{2}\right\rangle$ plotted against time, for the initial states $|8,2\rangle_{12}$ and $|9,2\rangle_{12}$. In both cases the minimum of $\left\langle\hat{n}_{2}\right\rangle$ is now 2. For the case when $M=10$, we see that at $t=1,\left\langle\hat{n}_{2}\right\rangle=8$, which is consistent with the state undergoing the transformation $|8,2\rangle_{12} \rightarrow|2,8\rangle_{12}$. When the initial state is $|9,2\rangle_{12}$ and thus $M=11,\left\langle\hat{n}_{2}\right\rangle$ is always less than 9 , and thus there is never a point where we transfer the 9 photons that were initially in the first mode, to the second mode.

The Hamiltonian (27) was designed so that it would perform the transformation $\mid N \pm$ $1,0\rangle_{12} \rightarrow|0, N \pm 1\rangle_{12}$, while leaving a state with exactly $N$ photons unchanged. It is clear that if our system is initially prepared in the state $|N, 0\rangle_{12}$, then the system will stay in that state. In fact, any state with exactly $N$ photons will remain unchanged under the action of (27). If our system has $N \pm 1$ photons then the dynamics of the Hamiltonian (27) will be identical to that of (22).

\section{Conclusions}

We have investigated a class of nonlinear Hamiltonians. These Hamiltonians were constructed from a simple beam splitter Hamiltonian, equation (11), and from the photon number operator $\hat{n}$. Due to the pivotal role played by the beam splitter Hamiltonian, (1), we investigated its properties thoroughly. The eigenvalues and eigenvectors of equation (11) were obtained using the theory of orthogonal polynomials. In particular, it was shown that the eigenvalue problem associated with equation (10) was equivalent to solving the three term recurrence relation associated with a class of orthogonal polynomials known as Krawtchouk polynomials. We were thus able to show that the dynamics of the beam splitter Hamiltonian and the class of nonlinear Hamiltonians, are exactly solvable using Krawtchouk polynomials.

Nonlinear Hamiltonians were then constructed from the beam splitter Hamiltonians and the total photon number operator. The dynamics of these Hamiltonians enabled novel tasks such as state swapping and conditional state swapping, to be performed. The formalism that we have introduced and the Hamiltonians that we have studied, have applications in quantum optics and quantum information. In particular, they can be used in the areas of state preparation and 


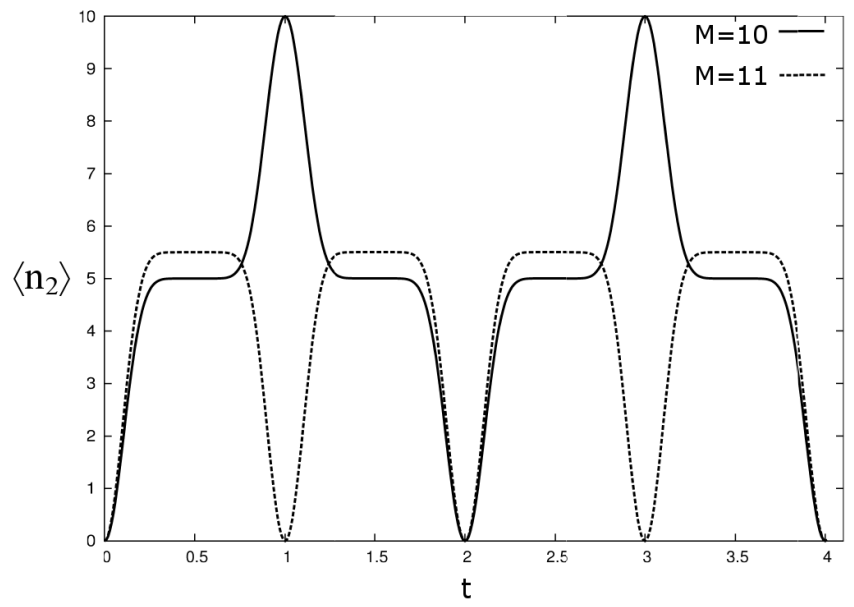

Figure 5: A plot of the expectation value $\left\langle\hat{n}_{2}\right\rangle$, against time, with $\gamma=\tau=1$ and where the initial state is $|M, 0\rangle_{12}$. It can be seen that for $M=11$ the Hamiltonian acts to trap some of the energy in the first mode, while for $M=10$ the energy is fully swapped between the two modes.
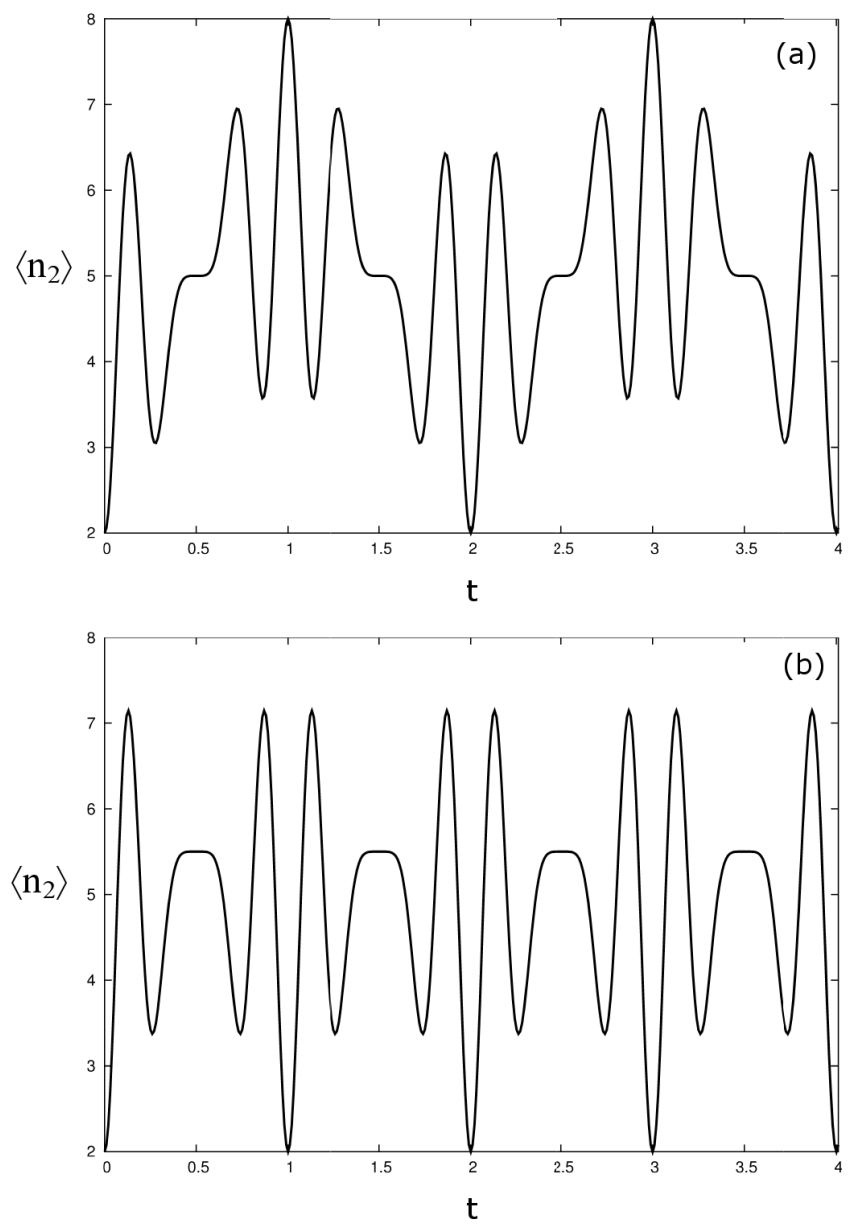

Figure 6: A plot of the expectation value $\left\langle\hat{n}_{2}\right\rangle$, against time, with $\gamma=\tau=1$. In plot (a) the initial state is $|8,2\rangle_{12}$, while in plot (b) the initial state is $|9,2\rangle_{12}$. 
in quantum control. For example, we have shown how the nonlinear Hamiltonian (23) can be used to prepare a superposition of Schrödinger cats states for a coherent state. In addition to this we have shown how we can control several modes so as to sort photons into one particular mode, where the mode that there are transferred to depends on the total number of photons. This was achieved by turning on couplings between two different modes, where the interactions were described using the nonlinear Hamiltonians (23) and (27). While we have discussed this work in terms of optics and photons, the results would apply to any system of bosonic particles.

\section{Acknowledgements}

We acknowledge financial support from grants MSM6840770039 and MSMT LC06002 of the Czech Republic. The authors are obliged prof. A. Odzijewicz for helpful comments and remarks.

\section{References}

[1] P. G. Kwiat, K. Mattle, H. Weinfurter, A. Zeilinger, A. V. Sergienko and Y. Shih Phys. Rev. Lett., 1995, 75, 4337-4341.

[2] S. M. Jensen, IEEE J. Quantum Elect., 1982, EQ-18, 1580-1583.

[3] A. Chefles and S. M. Barnett, J. Mod. Opt., 1996, 43, 709-727.

[4] G. J. Milburn and C. A. Holmes, Phys. Rev. Lett. 1986, 56, 2237-2240.

[5] B. Yurke and D. Stoler, Phys. Rev. Lett. 1986, 57, 13-16.

[6] A. Mecozzi and P. Tombesi, Phys. Rev. Lett. 1987, 58, 1055-1058.

[7] B. Yurke and D. Stoler, Phys. Rev. A 1987, 35, 4846-4849.

[8] R. Koekoek, R. F. Swarttouw, The Askey-scheme of hypergeometric orthogonal polynomials and its q-analogue, Report DU 98-17, TUDelft, 1998; http://aw.twi.tudelft.nl/ koekoek/askey.html

[9] M. Horowski, G. Chadzitaskos, A. Odzijewicz and A. Tereszkiewicz J. Phys. A, 2004, 37, 6115-6128.

[10] A. Odzijewicz, M. Horowski and A. Tereszkiewicz J. Phys. A2001, 34, 4353-4376.

[11] M. Horowski, A. Odzijewicz, and A. Tereszkiewicz Cz. J. Phys.2002, 52, 1231-1237.

[12] B. Yurke, S. L. McCall and J. R. Klauder, Phys. Rev. A,1986, 33, 4033-4054.

[13] R. A. Campos, B. E. A. Saleh and M. C. Teich, Phys. Rev. A, 1989, 40, 1371-1384.

[14] U. Leonhardt, Phys. Rev. A, 1993 48, 3265-3277.

[15] T. S. Chihara, An introduction to orthogonal polynomials, Gordon and Breach, New York, 1978.

[16] H. S. Wilf, Mathematics for Physical Science, Dover publishing, New York, 1962.

[17] J. Favard, Sur les polynomes de Tchebicheff, C. R. Acad. Sci. Paris Sr. I Math.1935, 200, 2052-2053.

[18] M. A. Nielsen and I. L. Chuang, Quantum Computation and Quantum Information, Cambridge University Press, Cambridge, 2000.

[19] S. M. Barnett and S. J. D. Phoenix Phys. Rev. A 1989, 40, 2404-2409.

[20] S. M. Barnett and S. J. D. Phoenix Phys. Rev. A 1991, 44, 535-545.

[21] R. Loudon, The Quantum Theory of Light, Oxford University Press, Oxford, 2000.

[22] J. R. Klauder and E. C. G. Sudarshan, Fundamentals of Quantum Optics, W. A. Benjamin, Inc., New York, Amsterdam, 1968.

[23] E. Schrödinger, Naturwissenschaften 1935, 23, 807-812. 